\title{
Evaluation of the Effectiveness of Health Education on Menopause Symptoms and Knowledge and Attitude in Terms of Menopause
}

\author{
Tugce Koyuncu ${ }^{1,2, *}$, Alaettin Unsal ${ }^{1}$, Didem Arslantas ${ }^{1}$ \\ ${ }^{1}$ Public Health Department, Faculty of Medicine, Eskisehir Osmangazi University, Eskisehir 26480, Turkey \\ ${ }^{2}$ Karsiyaka District Health Directorate, Karsiyaka, İzmir 35560, Turkey
}

\section{ARTICLE INFO}

\section{Article History}

Received 22 November 2017

Accepted 18 April 2018

Keywords

Health education

Intervention

Menopause

\begin{abstract}
This study was carried out to evaluate the effectiveness of health education on menopausal symptoms, knowledge, and attitudes about menopause. To evaluate the efficacy of a training program, information about menopausal symptoms, knowledge, and attitude toward menopause was collected before and after health education. After the health education intervention, it was determined that there was a decrease in the somatic and psychological subdimensions of the menopausal symptom evaluation scale and in the total score. Positive changes were observed in the levels of knowledge about menopause, and positive and negative emotional subscales of the menopause attitude assessment scale. It is concluded that health education is an effective method to positively change the perceived severity of menopausal symptoms and menopause-related level of knowledge and attitude.
\end{abstract}

(C) 2018 Atlantis Press International B.V.

\section{INTRODUCTION}

Menopause refers to the last menstrual hemorrhage and it is a natural process that all middle-aged women experience. Women without any changes in their menstrual cycles were evaluated as premenopausal, women with changes in their cycle or whose last menstrual hemorrhage was $\leq 12$ months previous were evaluated as perimenopausal, and women whose last menstrual hemorrhage without any cause such as pregnancy, breastfeeding or treatment was $>12$ months previous were evaluated as postmenopausal [1].

Most women enter the menopause between the ages of 45 and 54 years. Life expectancy has increased in the last 100 years due to demographic transformation, but the age of menopause has not changed. For this reason, women spend much of their lives in the postmenopausal period. Over time, the proportion of women in the postmenopausal period has also increased within the whole population. Menopause, which has an important place in a woman's life, has become one of the important women's health issues that needs to be considered in health service planning and presentation $[2,3]$.

Some of the major complaints during menopause are hot flashes, vaginal dryness, nervousness, and sleeping problems. Although the causes of these complaints are not fully known, they are generally thought to be caused by estrogen deficiency [2]. However, the fact that these complaints do not exist in every woman in the menopausal period, or that they are not the same for every woman, and that the response to the treatment is different, suggest that they

*Corresponding author. Email: tugce_koyuncu@outlook.com cannot be explained only by estrogen deficiency. In addition to estrogen deficiency, some of the factors thought to be influential in the appearance of these complaints are features such as knowledge, attitude, perception, and behavior related to menopause [4].

Knowledge is a prerequisite for the use of health services. Women need to be well informed about health issues so that they can have a say in health-care decisions concerning themselves and the protection of their health [5]. Health education intervention could be an alternative way for coping with menopause symptoms [6].

Attitude is the tendency to organize thoughts, feelings, and possible behaviors about individuals, places, events, or ideas. It is the tendency to initiate or perform a certain behavior. Individuals' personal lives and experiences that they have gained so far, as well as the society in which they live, determines their attitudes $[7,8]$.

Cultural and ethnic characteristics of the society are important factors that influence women's attitudes toward menopause and the meaning of being middle aged. Women's perception of menopausal life as a natural process or illness, and developments in their lives at middle age, influence their attitude toward menopause. In studies conducted on menopausal complaints, it has been reported that the attitudes of women toward menopause are an effective variable in the occurrence and severity of menopausal complaints. It is possible to reduce the frequency and severity of menopausal symptoms with education to improve the attitude toward menopause $[9,10]$.

This study was carried out to evaluate the effectiveness of health education on menopausal symptoms, and on knowledge and attitudes around menopause. 


\section{MATERIALS AND METHODS}

The study is an intervention study conducted on women living in the district center of Beylikova between September 1, 2013 and June 22,2015 . It was carried out to evaluate the effectiveness of health education on menopausal symptoms, knowledge, and attitudes around menopause. According to the data provided by the Turkish Statistical Institute on 2012 Address Based Population Registration System, the population of the district center is 3257, of which 1646 (50.5\%) are male and 1611 (49.5\%) are female. A total of 33.2\% $(n=545)$ of the female population in the district center are in the age group of 40-64 years and constitute the study participants [11].

No sampling methods were used in the study. The houses in the district center were visited one by one to reach the whole population. However, 82 women (15.0\%) did not agree to participate in the study and 153 women $(28.1 \%)$ were not at home during the data collection. A total of 310 women (56.9\%) agreed to participate in the pre-test.

Some sociodemographic and gynecological characteristics were collected from women who were able to be reached and who agreed to participate in the study ( 310 women). The height and weight of the women were measured. A pre-prepared training program was conducted in the study group which included no chronic disease, no smoking, no surgical menopause, no history of hormone replacement therapy, and who agreed to participate in the training program. A total of 59 women from the study group were reached and informed about the health problems that may occur during the menopausal period and methods for coping with them. In order to assess the effectiveness of this intervention, 54 of the informed women needed to be reached.

The training was conducted using methods such as audiovisual training tools, demonstration, and question-answer, taking adult education characteristics into consideration and using effective presentation methods. Meeting halls of Beylikova Hospital and district municipality were used for training. The training comprised three 30-minute sessions. Sessions were held by the author (T.K.) who is a medical doctor. The contents of the sessions were prepared in the light of the literature as follows. (1) The first session: the phase of getting acquainted with general information about menopause. (2) The second session: health problems that may arise in perimenopausal and postmenopausal periods. (3) The third session: methods to help prevent health problems that may arise in perimenopausal and postmenopausal periods and methods to cope with these problems.

After 30-45 days for evaluation of the effectiveness of the training, the women who participated in the activity were reevaluated at their homes using the same scales.

In order to conduct the study, approval was obtained with the decision of Eskisehir Osmangazi University Non-Pharmaceutical Ethics Committee for Clinical Investigations, dated July 12, 2013 and numbered 80558721/245. The study's data were collected in accordance with the Helsinki Declaration.

In accordance with the aim of the study, a questionnaire consisting of four sections was prepared using the literature $[5,12,13]$. In the first part of the questionnaire, some of the sociodemographic and gynecological characteristics of the women (age, marital status, education, family income and working status, smoking status, chronic illness history, pregnancy, birth and number of children, history of abortion, menopausal status, early menopause, and the use of hormone replacement therapy) were included. In addition to this, addresses and phone information of the women were also included to invite them to the training program. The second section included a menopausal symptoms assessment questionnaire; in the third section, menopause information evaluation form questions were included, and the fourth section included a menopausal attitude evaluation questionnaire.

While assessing the menopausal status of women, the definitions of the Stages of Reproductive Aging Workshop study [1] were utilized. Women without any changes in their menstrual cycle were evaluated as premenopausal, women with changes in their cycle or whose last menstrual hemorrhage was $\leq 12$ months previous were evaluated as perimenopausal, and women whose last menstrual hemorrhage was $>12$ months previous were evaluated as postmenopausal [1].

The presence and severity of menopausal symptoms in the study were assessed by the Menopause Rating Scale (MRS). The scale was developed in 1996 by the Berlin Epidemiology and Health Research Center [14], and in 2009, the validity and reliability of the Turkish version were made by Metintas et al. [12]. The MRS consists of 11 questions with a 5-point Likert-type scale, and is used to assess the situation of women in the past month. There are three subscales of the scale: somatic, psychological, and urogenital. The scores that can be taken from the somatic and psychological subscales range from 0 to 16 , and the scores that can be taken from the urogenital subdimension range from 0 to 12 . The sum of the scores obtained from the subdimensions gives the total score of the scale. The total score that can be taken from the scale ranges from 0 to 44 . As the scores and total points of all three subdimensions increase, the severity of the symptoms also increases.

In the study, the menopause knowledge evaluation form was prepared to measure the level of knowledge about women's general information about the menopausal period, health problems in this period, and ways to cope with these problems. The pre-test form, which consisted of 38 proposals, finally became 24 positive and 10 negative proposals, giving a total of 34 , after the content validity and reliability were calculated. The Cronbach $\alpha$-coefficient was 0.758 . The correlation between test-retest scores was moderate (rs: $0.630, p<0.001$ ). The answers to the suggestions in the scale were "right," "wrong," and "no idea." In evaluating the proposals, one point is given to the correct known proposal and zero points if there is no idea and misinformation. The total score of the scale is obtained by summing the scores of all the proposals arithmetically. The scores that can be taken from the scale range from 0 to 34 . As the score increases, the level of knowledge increases.

The Menopausal Attitude Assessment Scale (MAAS) was used to assess attitudes toward menopause. The validity and reliability study of the scale was performed by Koyuncu et al. [15]. The scale consists of four subscales; positive emotional (PE), negative emotional (NE), family relations (FR), and behavioral (B). The PE subscale consists of five proposals, including positive emotions about menopause. The PE subscale scores range from 0 to 20 . The NE subscale consists of three proposals including negative emotions about menopause. The points that can be taken from this subscale vary between 0 and 12 . The FR subscale includes three proposals about the relationship of women with their families during menopause. The points that can be taken from this subfield range 
from 0 to 12 . In the B subscale, there are two proposals evaluating the behavioral attitudes of women against menopause. The scores that can be taken from this subscale range from 0 to 8 . The responses to the proposals on the scale are prepared in the form of a 5-point Likert scale with the answers "I strongly agree," "I agree," "Undecided," "I disagree," and "I absolutely disagree." The points that can be taken from each proposal range from 0 to 4 ; 0 for the "absolutely disagree" answer, and 4 for the "strongly agree" answer. The responses of the negative proposals $(1,2,3,4,5,10,12$, and 13) are scored by reversing them. The increase in scores from the scale subscale indicates a positive attitude toward menopause.

The obtained data were evaluated on the computer using IBM SPSS version 20.0. The normal distribution suitability of the variables was assessed by the Shapiro-Wilk test. Wilcoxon paired two-sample tests were used to compare data before and after the training. Statistical significance was accepted as $p<0.05$.

\section{RESULTS}

The study group consisted of 54 women aged between 40 and 64 years, with a mean age of $50.41 \pm 6.36$ years. A total of 44 women $(81.5 \%)$ who constituted the study group were at primary school and lower educational level, and 40 (74.1\%) were housewives. Fifty $(92.6 \%)$ of the women were married. A total of $30(55.6 \%)$ of the women in the study group had four or more pregnancies, and $36(66.7 \%)$ had at least one miscarriage.

The mean menopausal age was $46.21 \pm 4.70$ years (range, 40.055.0 years). In the study group, the number of women in the premenopausal period was $12(22.2 \%)$, the number of women in the perimenopausal period was $14(25.9 \%)$, and the number of women in the postmenopausal period was $28(51.9 \%)$.

The median scores of MRS were 5 (range, 0-15) in the somatic subscale, 7 (range, 0-14) in the psychological subscale, 3 (range, 0-8) in the urogenital subscale, and 14.5 (range, 2-33) in the total score of the scale. The three most prevalent menopausal symptoms were physical and mental fatigue, joint and muscle disorders, and nervousness in the study group.

The scores of the study group in the menopause knowledge evaluation form ranged from 0 to 32 and the median score was 22.0.
The median scores of the women from the MAAS were 11 (range, $3-17$ ) in the PE subscale, 5 (range, 0-12) in the NE subscale, 8 (range, $0-12$ ) in the FR subscale, and 4 (range, $0-8$ ) in the B subscale.

After training, symptoms of menopause, knowledge level, and attitudes toward menopause were measured again with the same scale. The distribution of the scores of the women in the intervention group before and after informing about the scales is given in Table 1.

After the intervention, it was determined that there was a decrease in the somatic and psychological subscales of MRS and total score according to the total preeducation scores. No change was observed in the urogenital subdimension. Positive changes were observed in the levels of knowledge about menopause and the PE and NE subscales of the menopause attitude assessment scale. There were no differences in the scores of pre- and post-training in the FR and B subdimensions.

\section{DISCUSSION}

Symptoms in the menopausal period vary according to the characteristics of individuals and societies. The three most prevalent menopausal symptoms were physical and mental fatigue, joint and muscle disorders, and nervousness in the study group. Chou et al. [16] also reported sleep problems as the fourth most common symptom. Depressive mood was added to these symptoms in some studies [17-19]. Another study reported that vasomotor symptoms such as hot flashes are the most common in the menopausal period [20]. Among the reasons for the different outcomes reported in several studies, the differences in terms of sociodemographic, ethnic, genetic, and lifestyle-related features can be included. The fact that the diagnostic tools used in the studies are different can also cause the results to be distributed over such a wide range.

Having the right information about menopause can help women to have more realistic expectations about the menopausal period and to make better choices between treatment options for menopausal symptoms [21]. In health education for women in the menopausal period, the aim is to increase the knowledge about menopause in women and positively change the way menopausal symptoms are perceived [10]. In this study, the aim was to assess the effects of health education, including on menopausal symptoms and ways of coping

Table $1 \mid$ Distribution of median scores from the Menopause Rating Scale (MRS), the Menopause Knowledge Evaluation Form (MKEF), and the Menopausal Attitude Assessment Scale (MAAS) before and after intervention

\begin{tabular}{|c|c|c|c|c|c|}
\hline & \multicolumn{2}{|c|}{ Before intervention } & \multicolumn{2}{|c|}{ After intervention } & \multirow{2}{*}{$\mathrm{z} ; \boldsymbol{p}$} \\
\hline & Median & $25-75 \%$ & Median & $25-75 \%$ & \\
\hline \multicolumn{6}{|l|}{ MRS } \\
\hline Somatic & 5.00 & $3.00-8.25$ & 5.00 & $2.00-7.00$ & $2.740 ; 0.006$ \\
\hline Psychological & 7.00 & $4.00-10.00$ & 6.00 & $3.00-9.25$ & $1.986 ; 0.047$ \\
\hline Urogenital & 3.00 & $1.00-5.25$ & 3.00 & $1.00-5.00$ & $0.230 ; 0.818$ \\
\hline Total & 14.50 & $10.00-22.00$ & 14.00 & $7.75-21.00$ & $2.674 ; 0.007$ \\
\hline \multicolumn{6}{|l|}{ MKEF } \\
\hline Total score & 22.00 & $18.00-25.00$ & 27.00 & $20.00-29.50$ & $4.933 ; 0.000$ \\
\hline \multicolumn{6}{|l|}{ MAAS } \\
\hline Positive emotional & 11.00 & $8.00-13.00$ & 12.00 & $9.75-14.00$ & $2.620 ; 0.009$ \\
\hline Negative emotional & 5.00 & $3.00-7.00$ & 6.00 & $4.00-8.00$ & $2.980 ; 0.003$ \\
\hline Family relations & 8.00 & $5.75-9.00$ & 8.00 & $6.00-9.00$ & $0.246 ; 0.806$ \\
\hline Behavioral & 4.00 & $2.00-6.00$ & 5.00 & $3.00-6.00$ & $1.478 ; 0.140$ \\
\hline
\end{tabular}


with these symptoms, on women's symptoms of menopause, knowledge levels, and attitudes about menopause. It was determined that the frequency of menopausal symptoms in the subscales of women, except for urogenital subtype symptoms of MRS, decreased after intervention. In the urogenital subdimension, there was no difference before and after intervention. One of the reasons for this is that the symptoms (hot flashes, malaise, nervousness, etc.) questioned in the somatic and psychological subscale may be more subjective than the symptoms questioned in the urogenital subscale (urinary incontinence, vaginal dryness, etc.). There are studies reporting that the perceived severity of menopausal symptoms is diminished after the implementation of a planned training program [5,22-24]. Health education may help women to make more realistic assessments of their symptoms, to set realistic targets for menopausal symptoms, and to change the way menopausal symptoms are perceived.

Knowledge levels about menopausal symptoms and ways of coping with them are a critical for women to access health care in the menopausal period. It is unlikely that women without knowledge of the health services offered for the treatment of menopausal symptoms will reach these services. For this reason, it is very important to conduct health education studies in order to increase the level of knowledge about menopause. In some studies, it has been reported that women with menopausal health education have increased knowledge of menopause $[5,25]$. In this study, it was determined that after the intervention, the level of knowledge women have about menopause was increased according to the intervention.

Increased levels of knowledge about menopause and health education may not always lead to a change of attitude. However, menopausal process functioning, such as how it emerges, can help women to eliminate the feeling of uncertainty experienced during this period and their negative attitudes about menopause $[8,10]$. In some studies, it has been reported that women's attitudes toward menopause changed positively after health education intervention $[5,24,25]$. After the intervention, women's scores on the PE and NE subscales of the MAAS increased in a positive direction. There was no change in the FR and $\mathrm{B}$ subscales. The fact that the society in which the study is conducted is a more closed society with respect to family relations and living in the countryside, may have made it difficult to have a postintervention change in this subscale. One of the reasons why the B subscale does not change is that changing the behavioral element of the attitude is more difficult than changing the emotional and cognitive elements.

\section{CONCLUSION}

In order to cope with the symptoms seen in the menopausal period, the attitude toward menopause needs to be taken into consideration. Health education is an effective method to positively change the perceived severity of menopausal symptoms, menopausal knowledge level, and attitude. Doctors who provide primary health care may help to reduce the perceived severity of menopausal symptoms if they give more information about menopause to patients in the menopausal period. It may be useful to conduct a qualitative study to assess women's attitudes toward menopause in more detail.

\section{CONFLICTS OF INTEREST}

There is no conflicts of interest for all authors.

\section{REFERENCES}

[1] Soules MR, Sherman S, Parrott E, Rebar R, Santoro N, Utian W, et al. Executive summary: stages of reproductive aging workshop (STRAW). Climacteric 2001;4;267-72.

[2] Bruce D, Rymer J. Symptoms of the menopause. Best Pract Res Clin Obstet Gynaecol 2009;23;25-32.

[3] Hacettepe University Institute of Population Studies. 2013 Turkey Demographic and Health Survey. Ankara, Turkey: Hacettepe University Institute of Population Studies, T.R. Ministry of Development and TÜBİTAK; 2014.

[4] Utian WH. Psychosocial and socioeconomic burden of vasomotor symptoms in menopause: a comprehensive review. Health Qual Life Outcomes 2005;3;47.

[5] Tsao LI, Huang KE. Effectiveness of a perimenopausal health education intervention for mid-life women in northern Taiwan (\# MS03-21-LW). Patient Educ Couns 2004;54;321-28.

[6] Yazdkhasti M, Keshavarz M, Merghaati Khoei ES, Hosseini AF, Esmaeilzadeh S, Amiri Pebdani M, et al. The effect of support group method on quality of life in post-menopausal women. Iran J Public Health 2012;41;78-84.

[7] İnceoğlu M. Tutum Alg1 İletişim. 5th ed. İstanbul: Beykent University Publications; 2010, p. 13-29.

[8] Noroozi E, Dolatabadi NK, Eslami AA, Hassanzadeh A, Davari S. Knowledge and attitude toward menopause phenomenon among women aged 40-45 years. J Educ Health Promot 2013;2;25.

[9] Ayers B, Forshaw M, Hunter MS. The impact of attitudes towards the menopause on women's symptom experience: a systematic review. Maturitas 2010;65;28-36.

[10] Towey M, Bundy C, Cordingley L. Psychological and social interventions in the menopause. Curr Opin Obstet Gynecol 2006;18;413-17.

[11] Turkish Statistical Institute. Address Based Population Registration System Results. 2012. Available from: https://biruni.tuik.gov.tr/ medas $/$ ?kn=95\&locale $=\operatorname{tr}$ (accessed 15 November 2017).

[12] Metintas S, Arýkan I, Kalyoncu C, Ozalp S. Menopause Rating Scale as a screening tool in rural Turkey. Rural Remote Health. 2010;10;1-11.

[13] Neugarten BL, Wood V, Kraines RJ, Loomis B. Women's attitudes toward the menopause. In: The Meanings of Age: Selected Papers of Bernice L. Neugarten, Chicago: The University of Chicago Press; 1996, p. 193.

[14] Heinemann LA, Potthoff P, Schneider HP. International versions of the menopause rating scale (MRS). Health Qual Life Outcomes 2003;1;28.

[15] Koyuncu T, Unsal A, Arslantas D. Validity and reliability of menopause attitude assessment scale: a study in women aged 40-64 in Eskisehir-Mahmudiye. TAF Prev Med Bull $2015 ; 14 ; 448-52$.

[16] Chou MF, Wun YT, Pang SM. Menopausal symptoms and the Menopausal Rating Scale among midlife Chinese women in Macau, China. Women Health 2014;54;115-26.

[17] Elsabagh E, Abd Allah E. Menopausal symptoms and the quality of life among pre/post menopausal women from rural area in Zagazig city. Life Sci J 2012;9;283-91.

[18] Chedraui P, Pérez-López FR, Hidalgo L, Villacreses D, Domínguez A, Escobar GS, et al. Evaluation of the presence and severity of menopausal symptoms among postmenopausal women screened for the metabolic syndrome. Gynecol Endocrinol 2014;30;918-24. 
[19] Chedraui P, Aguirre W, Hidalgo L, Fayad L. Assessing menopausal symptoms among healthy middle aged women with the Menopause Rating Scale. Maturitas 2007;57;271-78.

[20] Ayranci U, Orsal O, Orsal O, Arslan G, Emeksiz DF. Menopause status and attitudes in a Turkish midlife female population: an epidemiological study. BMC Womens Health 2010;10;1.

[21] Naçar M, Baykan Z, Çetinkaya F. Perception and attitudes toward menopause: a study from Kayseri. Turkish J Pub Health 2009;6;36-45.

[22] Esposito Sorpreso IC, Laprano Vieira LH, Longoni Calió C, Abi Haidar M, Baracat EC, Soares JM Jr. Health education intervention in early and late postmenopausal Brazilian women. Climacteric 2012;15;573-80.
[23] Rotem M, Kushnir T, Levine R, Ehrenfeld M. A psychoeducational program for improving women's attitudes and coping with menopause symptoms. J Obstet Gynecol Neonatal Nurs 2005;34;233-40.

[24] Hunter M, O'Dea I. An evaluation of a health education intervention for mid-aged women: five year follow-up of effects upon knowledge, impact of menopause and health. Patient Educ Couns 1999;38;249-55.

[25] Elnaggar SA, Mohammed AH, El-R-Ibraheem SA. Health education effect on knowledge and attitude of peri-menopausal and menopausal women toward menopause at El-Arabin District in Suez Governorate. Life Sci J 2013;10;2838-46. 